\title{
Introduction to special issue: vascular co-option in cancer
}

\author{
Andrew C. Dudley ${ }^{1,2}$ \\ Published online: 4 December 2019 \\ (c) Springer Nature B.V. 2019
}

\section{Summary}

Like many great scientific ideas, the concept that solid tumors create new blood vessels that can be targeted as an anticancer strategy was met with skepticism and at times harsh criticism. Dr. Judah Folkman based his pioneering hypothesis on years of observations growing tumors in ex vivo perfused organs and later in the cornea of experimental animals. (The mammalian cornea does not contain blood vessels, so new blood vessels being induced by the presence of tumor-derived pro-angiogenic factors could easily be observed.) Next came studies using cultures of freshly isolated endothelial cells where biochemically purified angiogenesis inducers or inhibitors could be directly tested using in vitro bioassays. Eventually, the identification of potent angiogenic factors from tumor supernatants, for example, VEGF (vascular endothelial growth factor, and many others), informed preclinical models developing pharmacological inhibitors in allografted/xenografted tumors and in genetically engineered mice to formally test the hypothesis that targeting pro-angiogenic pathways could eradicate solid tumors. However, as is now well documented, clinical trials using angiogenesis inhibitors for various cancers have been met with disappointment, and this has spurred the generation of new hypotheses to account for the activation of complex resistance mechanisms that subvert the activity of anti-angiogenic therapies in human cancer patients.

When Folkman observed angiogenesis-dependent tumor growth in the rabbit cornea or in ex vivo perfused organs, alternative mechanisms for tumor neovascularization were either not known or not well understood. At the time, only a few studies had described potentially secondary yet

Andrew C. Dudley

acd2g@virginia.edu

1 Department of Microbiology, Immunology, and Cancer Biology, The University of Virginia, Charlottesville, VA 22908, USA

2 Emily Couric Cancer Center, The University of Virginia, Charlottesville, USA complementary modes of tumor blood vessel formation including seminal studies using incipient tumors engrafted in the hamster cheek pouch showing that primitive, cancer cell-lined networks could transport blood or fluid. These observations were perhaps a harbinger of the now well-documented phenomenon of vasculogenic mimicry which itself could be an important underlying contributor to the poor performance of anti-angiogenic therapies for various cancers. Similarly, cancer cells growing around established vasculature in a process termed "vascular co-option" may allow tumors to grow in a manner that does not depend on newly formed blood vessels, but instead takes advantage of preexisting ones. The concept of vascular co-option has recently received renewed attention and prominence due to the now well-documented role of vessel co-option in mediating resistance to vascular-directed therapies in human patients. In addition, vessel co-option (also known as "angiotropism" or "perivascular/extravascular migration") appears to play important roles in diverse processes related to cancer progression including dormancy, intra-organ dissemination, and immune evasion.

In this Special Issue of Angiogenesis, we have compiled six focused mini-reviews that cover specific aspects related to the concept of blood vessel co-option by cancer cells. Leading experts in the field have contributed: (i) GarciaGomez and Valiente (https://doi.org/10.1007/s10456-01909693-x) describe molecular mechanisms of vascular cooption during brain metastasis with an emphasis on the role of L1CAM-mediated dissemination, latency, and immune evasion. (ii) Seano and Jain (https://doi.org/10.1007/s1045 6-019-09691-z) contribute new insights into the role of vessel co-option in glioblastoma which is a highly lethal neoplasm where cancer cells are well known to migrate along the abluminal surface of the brain vasculature. Therapeutic strategies for targeting vessel co-option in glioblastoma are also discussed. (iii) Zhang and colleagues (https://doi. org/10.1007/s10456-019-09684-y) have comprehensively described the in vitro/in vivo models and cancer cell lines that are used to study vessel co-option, and they discuss the different molecular mechanisms that enable vessel 
co-option by cancer cells of different origins. (iv) Lugassy and colleagues (https://doi.org/10.1007/s10456-019-09695 -9) describe the phenomenon of "pericyte mimicry" and extravascular migratory metastasis by cancer cells and link these features to a recapitulation of embryonic-like developmental programs. (v) Vermeulen and colleagues (https ://doi.org/10.1007/s10456-019-09690-0) summarize key pathological features of vessel co-option versus sprouting angiogenesis and have done so based on tumor type, stage, or organ microenvironment. Relationships between frequency/presence of vessel co-option and patient outcomes are also discussed. (vi) Finally, Kuczynski and Reynolds (https://doi.org/10.1007/s10456-019-09698-6) summarize key studies demonstrating how vascular co-option mediates resistance to anti-angiogenic therapy and they add a new perspective on how vascular-targeted therapies could be used to inhibit co-option or augment the activity of, for example, immunomodulatory drugs.

In total, these reviews describe most, but certainly not all, of the key features that enable vascular co-option by cancer cells thereby contributing to cancer progression via diverse mechanisms. Reflecting on the work described herein, several open questions come to mind. For example, do cancer cells co-opt or migrate along the surface of lymphatic vessels using mechanisms similar to blood vessel co-option? How does vessel co-option relate to cancer dormancy and long-term survival of disseminated cancer cells following different treatment strategies? And can vascular co-option be selectively targeted to augment other therapies or prevent the dispersal of cancer cells further into organ microenvironments such as the brain following focused beam irradiation or chemotherapy? The six mini-reviews provided in this Special Issue will act as a framework for beginning to answer these and many other questions related to important roles of vascular co-option during cancer progression.

Publisher's Note Springer Nature remains neutral with regard to jurisdictional claims in published maps and institutional affiliations. 